Feminine-maternal traits, emotional stability, tolerance, and mature response to aggression or infantile conduct are among the assets to look for in nurses assigned to the care of excessive eaters and drinkers. Psychological studies offer guidelines for identifying suitable personality traits in candidates for nursing nutritional deviants.

\title{
Nurses for Alcoholics
}

\author{
By GIORGIO LOLLI, M.D.
}

$\mathrm{T}^{\mathrm{N}}$ HE number of hospital wards, sanatoriums, nursing homes, and clinics devoted to the care of alcoholics is growing steadily with the realization of the contemporary magnitude of this problem and the improved methods of diagnosis and treatment. At the same time the importance in our culture of other deviations in nutritional habits, such as obesity and leanness, is gaining increasing medical attention.

The establishment of facilities for the treatment of deviations in eating or drinking has naturally created a demand for qualified professional personnel. The personality traits of nurses to be assigned to services of this type are of particular consequence.

The serious responsibilities attendant upon ministering to the sick call for painstaking care in the selection and assignment of all nursing personnel. Hospital executives in the past had to rely, and not without considerable success, on their empirical experience and intuition for guidance in this function. The trend now, however, is toward application of sound and proved psychodynamic principles to the appraisal of nurse candidates. But still more can be achieved if selection is geared to the needs of treatment teams working with special classes of patients.

Dr. Lolli is research associate of applied physiology at Yale University, New Haven, Conn.
My discussion, therefore, centers on the importance of the emotional makeup of nurses to be assigned to a service devoted to the correction of deviant eating and drinking habits in which the approach is simultaneously medical, psychological, and social.

The extension of these principles to other types of clinical services, it is hoped, will be self-evident.

The emphasis on interactions between the nurse and the alcoholic patient should be construed only as an expression of the belief that the nurse who is able to deal adequately with the alcoholic can also deal appropriately with all patients regardless of the condition for which they need nursing care. When sick, all individuals to a certain extent revert to infantile patterns of behavior. The understanding and handling of this behavior is facilitated by knowledge about alcoholism-a condition which results from the eruption of infantile traits in adult age.

Finally, it is assumed that the importance of the nurse's professional qualifications is universally granted and needs no further emphasis here.

The suggested criteria represent an ideal conception of the nurse qualified to work in a service for eating and drinking deviants. But even if ideals seldom, if ever, can be attained in full, our constant goal should be to approach the model.

A systematic evaluation of the emotional as- 
sets and liabilities of the nurse prior to her assignment to a particular service will weed out some candidates who would affect and be affected unfavorably by the institutional group. Of course, evaluation must continue after the nurse becomes a member of the staff. It should be an endless and creative process of mutual criticism wherein the appraisal of the newcomer by the older members is not more important than her appraisal of the group. Final decisions about long-term employability should be taken only after several months of trial.

Certainly nurses, like other members of the human race, cannot be expected to be without emotional inadequacies. However, some difficulties which are not necessarily handicaps in other professions or occupations might present a serious threat to a satisfactory nurse-patient relationship.

The emotional problems which represent a handicap in a nurse assigned to a service for deviant eating and drinking habits usually are deeply rooted and difficult to alleviate or solve. The illusion that personality changes may occur as a result of learning experiences with staff members or with patients should not be fostered. In a service of this type, time is seldom available for entirely adequate treatment of the patient. 'Time is never available for more than a superficial approach to the emotional difficulties of members of the staff. On the other hand, emotional problems of the staff may be swiftly magnified, or change from latency into open manifestations, as a result of exposure to an emotionally disturbing environment.

In the selection and evaluation of nurses, the concept of "compatible neuroses" is a practical one. When a new nurse is employed the composition of the staff as a whole should be kept in mind. It is desirable to visualize what effects the new member of the team will have on the older ones. For instance, some obvious handicaps in a nurse supervisor can be tolerated if other members of the team do not exhibit similar traits or are not too sensitive about them.

Excessive drinking, excessive eating, and deviant nutritional habits in general are linked with the very dawn of emotional life. Through the first nutritional experiences the first social experiences of the adult-to-be take place. Adequate hunger-linked emotions are those of love, warmth, and mutual tenderness. The inadequate ones are those of hate, violence, and resentments in general. A mutually rewarding mother-child relation favors the development of adequate hunger-linked emotions. Conversely, an unsatisfactory mother-child relation has untoward repercussions unless outweighed by later and more farorable experiences.

When psychological and social techniques are applied to problems of nutrition and nutritional habits, uncontrollable emotions often flare up quickly and violently not only in patients but also in emotionally ill-equipped therapists. The reason for this is that the emotions linked with deviant nutritional habits are antisocial in their nature. Indeed, it may be that unconscious fears related to these emotions account for the paucity of attention given thus far to the psychological and social aspects of hunger and of nutritional habits in general.

\section{Physical Characteristics}

In a general hospital, the nursing staff of a service for nutritional deviants should be limited to women. A general hospital has male attendants available if necessary for security. Experience has shown that with properly trained and emotionally fit women nurses, with medication administered promptly and adequately, and with a response to violence which emphasizes firm and fearless tolerance, the need for restraints and for the use of force in general is practically nil.

The limitation to women nurses is based on the crucial role played by women in bringing up children, as known from time immemorial and confirmed by scientific research during recent decades. While unsatisfactory motherchild relations contribute to all cases of adult maladjustment, they are especially decisive in those directly linked to deviant nutritional habits. Conversely, healthy emotional experiences with adequate women may help to alleviate or wipe out the painful pressure of half-buried experiences with the woman who is of paramount importance in the life of every human being. This stress on the significance of an unhealthy mother-child relationship should not be construed as "criticism" of the mothers of 
patients or of the patients themselves. It is simply a recognition of the fact that inadequacies of the mother-child team have farreaching consequences.

The marital status of the nurse seems unimportant. There are countless single women who display clear-cut feminine personality characteristics, objective attitudes toward sexual behavior and its deviations, and ability to participate in a variety of mutually rewarding social relations. Because healthy attitudes toward sex problems are often entirely unrelated to sex experience in real life, inquiries about sex life are seldom important for evaluation. Such inquiries may rather be symptomatic of the problems of the interviewer than evocative of those of the candidate.

It follows that marriage or sex experience does not make feminine a woman who is not so from the start; nor will either or both solve sexual problems. The presence or absence of sexual difficulties stems psychodynamically from the presence or absence of maladjustment in the area of hunger-linked emotions. This is why problems connected with distortions of the ability to love, to relate to people, to be tender, to express warm feelings appear to be of fundamental significance.

The age of the candidate is of secondary importance, provided she is in excellent physical health.

\section{Shared Handicaps}

In a service devoted to deviant eating and drinking habits, excessive drinking and marked overweight or underweight represent obvious handicaps. Moderate overweight or underweight can be tolerated if the nurse is able to accept possible criticism from patients about her "weakness" and to use the handicap as a veiled indication that she is "not different." One of the main difficulties of the neurotic patient is his feeling that he is "different." $\mathrm{He}$ sometimes feels encouraged by seeing that other people who have achieved some measure of success in life also exhibit handicaps similar to his own.

If her language is clearly intelligible, a foreign accent should not disqualify a candidate. In many cases, the neurotic patient feels him- self a "stranger" or "estranged." Contact with another "stranger" may be emotionally helpful. People sometimes share with a traveling companion, a stranger, facts and feelings which they could not share with a close friend.

The ability to express herself clearly in writing, however, is important for the nurse because of the great value of her observations, which should be recorded in detail.

With these possible exceptions, however, it must be remembered that in a professional nurse-patient relationship the emphasis should be on shared assets rather than on shared handicaps.

All that has been said thus far in no way attenuates the importance of basic professional and technical training. In addition, some knowledge or at least some specific interest in nutrition and dietetics is desirable since the attitude of the deviant patient toward food often supplies revealing clues to his emotional life.

\section{Psychodynamic Principles}

It is advisable that a candidate be interviewed separately by two members of the staff whose training and experience allow them to base an evaluation of the nurse's assets and liabilities on sound psychodynamic principles.

The following questions may be considered as a general guide for interviewers but are not intended to be used as an interview outline.

Has the candidate ever had any problem connected with the excessive use of alcohol or drugs? If so, a thorough psychiatric evaluation should be conducted before a decision is taken, even if the addiction to alcohol or drugs is no longer active. The rule that persons presently addicted to alcohol or drugs should not be considered for a position in a service specializing in eating and drinking deviations has no exceptions. For persons who were addicted to alcohol or drugs in the past, this rule could have some exceptions which only a thorough personality study of the candidate may justify.

The emotions linked with excessive use of alcohol or of drugs and those linked with nutritional deviations in general tend to be explosive. These are the emotions whose distortions lead to a variety of serious individual and social disorders, besides alcoholism or drug addiction. 
Persons suffering from these emotional turmoils often have their problems fanned by exposure to patients suffering from similarly distorted emotional constellations.

The experience of Alcoholics Anonymous, an association of former excessive drinkers who help each other to keep sober, might seem to contradict this viewpoint. $\Lambda$ deeper examination of the difference between a therapeutic relationship with patients and fellowship in Alcoholics Anonymous will prove that no such contradiction exists.

The attitude toward Alcoholics Anonymous of any service dealing with alcoholic patients should be friendly and cooperative, giving full recognition to the great contribution of this movement to the alleviation of the problem of alcoholism.

In most cases the professional therapeutic approach to the alcoholic patient is compatible with the activities of Alcoholics Anonymous. Thus, with exceptions based on psychiatric contraindications, the alcoholic patient should be encouraged to participate in the movement. Nevertheless, the fundamental differences between a professional and a nonprofessional approach must never be lost to sight. It is true that all professional as well as lay methods used to control alcoholisin are based on the same process: Emotional energies tied to physical disability, psychological inadequacies, and social maladjustment, or a combination of these disorders, are liberated through the alleviation or correction of the handicaps and then used to keep the urge to drink in check. But professional approaches and the Alcoholics Anonymous method differ quite markedly in the degrees of alleviation and correction of the medico-psycho-social handicaps and even more in the techniques of achieving such alleviation or correction.

The members of a professionally trained treatment team should approach the alcoholic, as all patients, on the fertile ground of common humanity. The lines of communication with him are tacitly sustained by the assets shared with him. While the therapists take their own liabilities for granted, they do not share them with the alcoholic beyond the implicit admission of their existence.

To try to cooperate with the alcoholic on a professional level calls for a variety of assets in the nurse. She must be able to direct an abundant, flexible, and timely flow of emotions to those areas of her personality which are challenged by the alcoholic's difficulties. For example, there is need for large emotional reserves so that the response to aggression will be tolerance, so that the wounds inflicted by hate will be healed without excessive challenge to the nurse's proper attitude. In the ideal attitude, the nurse's wishes and her ethical duties should coincide.

Emotional reserves are not ample in the alcoholic. The nurse addict is no exception even if her abstention from alcoholic beverages has been of reasonably long duration, because she needs these emotions to keep in check her own addictive urge. This situation makes it difficult for her to deal with alcoholics on a professional basis.

The alcoholic nurse, like any other addict, is able to deal with and help her fellow alcoholics on a nonprofessional basis. This is shown decisively by repeated experience in Alcoholics Anonymous, where the line of therapeutic communication between members is sustained by the shared conscious experience of a handicap (namely, addictive drinking) and by the shared unconscious experience of those guilt-laden emotions which lead to addiction. The fact is that less emotional energy is required by the alcoholic to abstain in Alcoholics Anonymous than to refrain from drinking in response to professional treatment. The more economical achievements of Alcoholics Anonymous result from the circumstance that within the framework of the movement the addictive urge to drink is kept in check by mutually potentiating group emotions whose pressure is concentrated intensely-sometimes violentlyon the interplay of the two opposites: inebriety and sobriety.

A sound and adequate professional approach, on the other hand, leads to an ampler expansion and to a greater flexibility of the alcoholic's personality. The outcome is deeper : a less rigid acceptance of life in general and a more secure attitude toward the lifelong liability connected with the alcoholic problem in particular.

As already noted, professional and Alcoholics Anonymous approaches are compatible and 
their combination is advisable in many cases of alcoholism. In combined approaches, the abstention from alcohol achieved and maintained in Alcoholics Anonymous does not drain the alcoholic's emotions drastically and enables him to face a professional approach with greater chances of personal improvement.

But this fact must be borne in mind: While the alcoholic can "receive" profitably a combination of professional and nonprofessional help, only exceptional persons can give professional and nonprofessional help to the alcoholic at the same time. Yet this is the dangerous situation faced by a professional nurse who is an alcoholic in working with alcoholics as patients.

Whether or not she is a member of Alcoholics Anonymous, she is bound to share her liability with the alcoholics she nurses, either on a conscious or on an unconscious level. From this viewpoint, therefore, her line of communication with alcoholics is not professional. Yet in nursing duty she must try to establish a professional line of communication which, because based mostly on personal assets, calls for large amounts of free emotional energies. Repeated observation indicates that the nurse's attempt to reconcile professional and nonprofessional attitudes has a high liability to failure. In all likelihood, this attempt adds a new conflict to the old ones, with renewed drain on the nurse's emotional reserves. When dutybound to act professionally, either her defenses against the urge to drink weaken or an ill-concealed nonprofessional attitude takes the place of professional nursing. Dangers then arise for the alcoholic nurse as well as for the alcoholic being nursed.

The following propositions, based on fundamental principles of mental economics, summarize the above discussion:

1. In Alcoholics Anonymous and in some other lay approaches, the individual member gives the alcoholic as much help as he receives. The success of treatment is based on this equilibrium.

2. In a professional relationship, the therapist must be able and willing to give much and to receive nothing. The maintenance of the emotional equilibrium of professional persomnel is the outcome of interactions between these indi- viduals and society at large, with resulting availability of large emotional reserves for treatment purposes.

3. An alcoholic nurse, even if long sober, almost constantly faces difficulties in tolerating a situation of disturbed equilibrium in which the help she should give far exceeds what she receives.

These statements should not be construed as an attempt to demonstrate the superiority of professional treatment and professional personnel. Their aim is solely to clarify the differences between professional and lay approaches.

What is the candidate's attitude toward the emotional problems of patients? Extremes of excess interest and disinterest are equally undesirable. The adequate nurse seldom tries to elicit from patients conversation relating to emotions. However, patients often talk to her. She should be able to interrupt "confessions" which, almost intuitively, she senses to be damaging to the patient's emotional stability, insofar as the patient will later regret disclosures made in a state of turmoil.

The emphasis on emotional problems, so obvious in contemporary societies, can lead to an attitude characterized by almost total denial of body illness. The "it's all in your mind" type of nurse is not particularly helpful to patients. It hints of a grandiose and rather primitive conception of the powers of the human mind. To be aware that body and mind interact does not mean that disturbed emotions are the exclusive cause of body disorders, and certainly the nurse should not promote such an impression.

Is the candidate interested in bedside nursing? Some nurses are attracted by the scientific aspects of nursing, by theoretical discussions, and by administrative functions only because they are basically inhibited with patients. They are unable to be close to them, touch them, and so forth. Such attitudes are indicative of emotional problems which, related as they are to underlying deriant nutritional habits, are not compatible with the proper nursing of patients on a service specializing in these very problems.

Does the candidate have strong feelings of inferiority (or superiority) about the practice of nursing in comparison with the practice of internal medicine, or psychiatry, or social work? 
An unrealistic hierarchical conception of professions is the symptom of unsolved intellectual and, sometimes, emotional problems. The welltrained nurse has her rightful place in a therapeutic team on an equal basis. The best qualified nurse understands the nature of this equality.

Does the candidate believe that there are higher or lower activities in nursing? The good nurse has no such illusions. The handling of a bedpan is not an activity of a "lower" quality than some administrative functions. The quality of a function is determined by the training brought to it and by the emotional attitude toward it. Strivings for power which are incompatible with ministering to the sick often underlie concepts relating to higher or lower activities in nursing and in professions in general.

Has the candidate ever worked in close cooperation with social workers? If so, has she gotten along well with them? How does she feel about social workers in general? If she has not teamed with social workers in the past, is the likelihood of good cooperation apparent?

The profession of social work has gone through changes as radical as those observed in the profession of nursing in recent years. From the handling of some apparently simple social problems, social workers have shifted their attention in part to the emotional problems of the individual. The experience thus gained has deepened their insights into the social maladjustments which are the projection of inner inadequacies. Experience has shown that an approach to social difficulties based on sound psychodynamic principles can supply to the disturbed person those corrective emotional experiences which may affect his inner equilibrium favorably and durably.

The cooperation between nurse and social worker is most helpful to any patient in the hospital and outside. In the eyes of the alcoholic, for example, the nurse often represents the temporary shelter and security of an unreal world. The social worker, on the other hand, represents the harsh realities of day-by-day life. This seems to be one reason why so many hostilities are still polarized against the social worker. The emotional ambivalence toward maternal figures which governs the lives of so many neurotic individuals, alcoholics among them, often expresses itself with love for the nurse and hostility to the social worker. The nurse is often in a position to interpret the social worker to the alcoholic. Furthermore, the nurse, exposed more than any other professional person to the exhausting challenges of the alcoholic, often needs the emotional support, as well as the technical interpretation of the nurse-alcoholic relationship, which a skilled psychiatric social worker can supply.

How does the candidate envisage the specific role of the nurse in the prevention or alleviation of the emotional diffculties of patients? There is growing psychodynamic evidence that the main factor underlying emotional stability is a healthy and mutually rewarding mother. child relation. Regardless of her age, the nurse plays a maternal role for all patients and should be endowed with maternal traits. According to this viewpoint, maternal traits should be considered almost identical with feminine traits, and they are not dependent on the actual experience of motherhood. They are to be understood as the ability to tolerate and to interrupt betimes other people's pleasures and also their pains. The good mother is the one who knows how and when relief should be given to the child's pain; she knows also how and when the child's pleasures should be interrupted. In other words, she should be able to tolerate some pain in her child without guilt feelings and some pleasure without envious feelings. Besides these traits, emotional and physical stability, plus predictability of reactions to a given situation, should be considered maternal traits.

Does the candidate prefer male or female patients? There is little doubt that the ideal nurse should be maternal enough not to have preferences for male or female patients.

How would the candidate react to attempts by patients to get information about her per. sonal life? The ability not to disclose too much about her personal life is of great importance. Usually it is linked with the ability to give some 
harmless information about herself. In her contacts with the patient, the nurse should be able to keep control of the questions asked of her. This ability is definitely linked with her respect for other people and with her selfrespect.

What is the candidate's attitude toward success or failure? Persons whose emotional constellations are similar to those operative in alcoholism and in other deviant nutritional habits often have a kind of "all or nothing" attitude toward success and failure. A minor error represents total failure and, conversely, a minor achievement might be fantastically magnified into a major success. The ability to see these matters in their proper perspective is best suited to the nursing of patients of this type.

How does the candidate react to aggression? Completely passive and completely aggressive reactions are equally undesirable if present all the time. Of course, occasional passivity or aggression are part and parcel of human nature. When required by the welfare of the patient, a nurse should be able to enforce discipline without experiencing guilt. Her actions should be guided by reason and not precipitated by the overwhelming pressure of her aggressive emotions.

Does the candidate exhibit harmless infantile traits? The ability to be a child occasionally and to interrupt the often painful performances of adult behavior should not be considered a handicap. Brief expressions of harmless infantile traits may greatly help a nurse to control those infantile traits which are harmful. The upbringing of many disturbed adults, among them the alcoholics, was highlighted by parental or cultural attitudes pointing to natural childhood behavior as basically "wrong" and leading to repression of harmless infantile traits.

What is the candidate's attitude toward hierarchical transmissions of authority and nonphysical violence from top to bottom? There is little doubt that authority and violence are often, even though wrongly, linked together and that retaliation for authoritarian attitudes of superiors is meted out to persons inferior in rank and, eventually, to patients.
The ideal nurse should be able to block violence at her level and to act more as a buffer than as a catalyst.

Can the candidate distinguish between a friendly attitude toward a patient and being "friends" with the patient? Can she keep sufficiently close to him and at the same time sufficiently distant from him? In a service for deviant eating and drinking habits, many patients are in good physical condition 2 or 3 days after their admission. They tend to become "friends." They attempt to engage the nurse in a close relationship which is dangerous both to them and to the nurse. The latter must have the skill to maintain the relationship on a professional level.

Does the candidate apply the disease concept to alcoholism and obesity? The alcoholic and the obese are suffering as a result of their search for excessive and forbidden pleasures. The disease connotation of their predicament is unusual insofar as the concept of disease is customarily linked with the concept of pain. Sooner or later persons in contact with alcoholics and obese individuals sense - consciously or unconsciously--that a search for forbidden and excessive pleasures, much more than overwhelming pain, is at the root of the trouble. Some professional people, nurses included, cannot accept without resentment the excessive pleasures of the addicts and react with illdisguised hostility to the realities of the situation.

Is the candidate tolerant of the patient's lack of cleantiness? Alcoholics, especially in the early days of their hospitalization, are often unclean and unwilling to be clean. Sometimes this attitude is followed by an obsessive search for cleanliness. There is a type of woman, observed often in our culture, who was brought up with an obsessive repulsion against all that is not perfectly clean. She might feel "soiled" by the sexual experiences of marriage, and as a young mother she might be repelled by the soiled diapers of her baby. While this is not the place to discuss how and why this attitude develops, it can be stated that a rather tolerant attitude toward lack of cleanliness and an equally tolerant attempt to correct it are es- 
sential in the nurse assigned to patients on a service for nutritional deviants.

Is the candidate sensitive enough to the emergent needs of the neurotic to be able to satisfy them quickly if required? Is she able to interrupt his pain swiftly when it appears to be overwhelming? On the other hand, is she free enough of impulsive traits to be able to delay the interruption of pain when it seems justified to do so in terms of long-range therapeutic plans? The attitudes explored by these questions are linked with the previously discussed maternal traits.

Is the candidate so secure as to be able to discuss her inadequacies with colleagues and superiors? If unable to cope with a given situation or with a given patient, is she able to delegate the task to a colleague? These admissions of individual shortcomings can occur only within a well-integrated and smoothly operating therapeutic team, where mutual respect is based on technical competence but even more on high standards of morality and of emotional adequacy.

Can the candidate correctly interpret sexual or apparently sexual proposals by patients? There is little doubt that some patients react to nurses with the most childish side of their personalities. Sometimes they try to touch them, eager for a physical contact which is more that of the child with the mother than of the man or woman with another adult woman or man. The correct interpretation of these attitudes is fundamental and should lead to tolerance not free from firmness.

Is the candidate willing and able to give the patient all the explanations necessary to make clear the apparently mysterious ways in which a service of this type operates? Is she a good listener? Because of the attempt to elicit corrective emotional experiences, the attitude of the treatment team often differs radically from all attitudes to which patients had been exposed in the past. Thus the service and the actions of its personnel are often interpreted as puzzling and mysterious. Nurses should have the ability to clarify misunderstandings and define treatment goals. They should be able to listen with poise to the patient's excessive praise or criticism of other members of the therapeutic team, and be free to convey to colleagues and superiors the patient's feelings abcut them so that treatment may be modified accordingly.

\section{Summary}

Services devoted to patients with deviant eating and drinking habits, separately or in combination, are growing in number. The professional nurse is a most important member of the therapeutic team in such services. Available are psychodynamic principles which should be applied to the evaluation and selection of nurse candidates for services of this type.

The specific criteria suggested are intended to help in identifying both suitable and unsuitable emotional traits in the nurse seeking assignment to such a service. The chief guiding principle is that the nurse should be a woman able to function as a symbolic femininematernal figure. This is based on the observation that patients with deviant eating and drinking habits usually experienced some disturbance in the early mother-child relationship, with resulting distortion of hunger-linked emotions, and that this maladjustment can be alleviated through healthy emotional contacts with adequate woman figures.

Among the most desirable specific characteristics, therefore, are: stable emotional makeup; feminine-maternal traits (not dependent on age or sexual experience) ; ability to cooperate in a treatment team with social workers particularly; acceptance of the patient's condition, in spite of its origin in pleasureseeking activities (eating, drinking), as one requiring professional treatment; and ability to respond to the patient's occasional need for immediate relief from pain, or occasional aggressive and infantile conduct, without counter aggression and with appropriate mature behavior.

Nurse candidates who themselves have experienced addiction to alcohol or drugs require special psychiatric evaluation. 